Cahiers québécois de démographie

\title{
Des fiches de famille à la mesure des migrations
}

Une méthode élaborée à partir des données du Saguenay au $\mathrm{XIX}^{\mathrm{e}}$ siècle

\section{Measuring Migration from Family Files}

A Method Based on Data for the Saguenay Region in the $19^{\text {th }}$

Century

Medir las migraciones utilizándo las fichas de familia

Un método elaborado a partir de datos del Saguenay en el siglo XIX.

\section{René Jetté et Danielle Gauvreau}

Volume 16, numéro 1, avril 1987

URI : https://id.erudit.org/iderudit/600607ar

DOI : https://doi.org/10.7202/600607ar

Aller au sommaire du numéro

Éditeur(s)

Association des démographes du Québec

ISSN

0380-1721 (imprimé)

1705-1495 (numérique)

Découvrir la revue

Citer cet article

Jetté, R. \& Gauvreau, D. (1987). Des fiches de famille à la mesure des migrations : une méthode élaborée à partir des données du Saguenay au $\mathrm{XIX}^{\mathrm{e}}$ siècle. Cahiers québécois de démographie, 16(1), 37-65.

https://doi.org/10.7202/600607ar
Résumé de l'article

Il n'est pas facile de mesurer ni de caractériser les mouvements migratoires, encore moins quand ils concernent les populations du passé. Le présent texte propose à cet effet une méthode inédite fondée sur les familles reconstituées à partir des registres paroissiaux saguenayens. Utilisant les biographies individuelles et familiales, cette méthode permet d'étudier séparément les mouvements d'immigration et d'émigration. Dans le cas du Saguenay, et probablement de toute autre région à fécondité et mortalité élevées, elle permet par ailleurs de fixer la date des migrations à un moment peu éloigné de la première ou de la dernière apparition dans le fichier des familles. 
Cahiers québécois de démographie

Vol. 16, no 1, avril 1987

\title{
Des fiches de famille à la mesure des migrations: une méthode élaborée à partir des données du Saguenay au XIXe siècle
}

\author{
René JETTÉ* et Danielle GAUVREAU**
}

\section{INTRODUCTION}

Depuis plus de trois siècles, au Québec comme dans l'ensemble de l'Occident, les registres paroissiaux ou d'état civil servent à l'enregistrement régulier et obligatoire des naissances, des mariages et des décès. A condition d'avoir été tenus et conservés, ces documents officiels permettent aux démographes de mesurer le mouvement naturel de la population concernée.

A de rares exceptions près, comme celle des registres de population en Belgique (voir par exemple Watkins et Gutman, 1983), rien de tel n'existe toutefois pour mesurer le mouvement migratoire. En effet, aucune réglementation ne régit l'enregistrement des déplacements des personnes. L'ampleur de l'immigration et de l'émigration n'est donc évaluée qu'approximativement, par des méthodes plus ou moins satisfaisantes. Une première façon consiste à établir des bilans migratoires nets qui reposent sur des données d'état civil encadrées par deux recensements. Elle présente des limites qui sont surtout liées au fait qu'elle mesure des résultats nets, qui demeurent muets sur $1^{\prime}$ ampleur respective de 1 'immigration et de l'émigration.

Il est également possible de mesurer plus directement 1 'ampleur des entrées et des sorties en comparant des listes nominatives recueillies à divers moments (recensements, "city directories", etc.) pour y suivre les individus sédentaires et identifier ceux qui migrent, qu'il s'agisse d'immigrants ou

* CEGEP de Saint-Hyacinthe.

** Université du Québec à Chicoutimi, SOREP. 
d'émigrants. Le recours à une telle méthode est cependant rendu d'autant plus difficile que la période qui sépare deux listes est longue, les sorties d'observation étant alors plus nombreuses et d'origines variées. Les variations nominatives, qui rendent incertaine l'identification d'un nombre non négligeable de personnes et conduisent à une surestimation des mouvements migratoires, constituent un autre type de problème qui paraît non moins négligeable (Parkerson, 1982).

L'objet de cet article est de proposer une méthode de mesure des migrations dont l'originalité et la force résident dans le fait qu'elle s'appuie sur l'utilisation d'un fichier de familles reconstituées à partir des registres paroissiaux ${ }^{1}$. En effet, bien que les événements d'état civil (naissances, mariages, décès) et les événements migratoires (entrées, sorties) soient des événements relativement indépendants les uns des autres, il s'avère que les informations contenues dans les premiers peuvent constituer des indicateurs des trajectoires migratoires. Ainsi, I'occurrence même d'un événement et la déclaration du lieu de résidence des personnes qui en sont les sujets, attestent la présence de personnes dans un lieu et à un moment donnés. A l'inverse, un arrêt brusque de l'enregistrement de tels événements dans ce lieu peut, sous certaines hypothèses, être interprété comme le signe d'une émigration de l'ensemble ou de certains membres du groupe familial.

Le premier volet de la méthode présentée dans cet article consiste à définir le statut migratoire des personnes apparaissant dans les familles reconstituées. Le second consiste à établir le plus précisément possible, pour les personnes migrantes, le moment de l'entrée ou celui de la sortie. Puisque les mentions tirées des registres paroissiaux ne correspondent pas, en tant que telles, à des dates de migration, il faut alors répondre aux deux questions suivantes :

1. A notre connaissance, seule Craig (1983) a explicitement utilisé une telle approche pour saisir le phénomène migratoire dans la région de Madawaska. Par aflleurs, l'unique analyse méthodologique qui s'en rapproche a été menée par Jetté (1980). Dans le contexte particulier de la Nouvelle-France de la fin du XVIle siècle, il a posé les bases d'une méthode de reconstitution de recensements à partir de fichiers de population constitués sur la base de registres paroissiaux. Le phénomène migratoire n'a toutefois pas pu être isolé, étant donné que les effets de la migration sur l'histoire des individus se confondaient avec les effets des défectuosités des registres paroissiaux utilisés (lacunes dans les séries et sous-enregistrement d'événements, les décès notamment). 
- Quand un individu est mentionné pour la première fois dans les registres paroissiaux du territoire concerné, à une date quelconque postérieure à sa naissance, quelle durée sépare le moment de son immigration et celui de sa première mention?

- Quand un individu est mentionné pour la dernière fois dans les registres paroissiaux du territoire concerné, à une date quelconque antérieure à son décès, quelle durée sépare la date de cette dernière mention et celle de son émigration?

I1 est d'autant plus important d'apporter une réponse à ces deux questions que nous $n^{\prime}$ avons actuellement aucune idée de l'ampleur de cette durée, que ce soit de façon générale ou en fonction de caractéristiques spécifiques.

Avant d'aborder la définition proprement dite des statuts migratoires et la détermination du moment des migrations, nous présentons la nature générale des informations sur lesquelles s'appuie l'élaboration de cette méthode et plus spécifiquement le corpus de données saguenayennes utilisées ici. La dernière partie consistera en une épreuve de validation des règles précédentes à 1 'aide des recensements saguenayens.

\section{L'ELABORATION DE LA MÉTHODE}

\subsection{La nature générale des informations utilisées}

La mesure des migrations à partir de registres paroissiaux repose sur un mode d'observation relativement nouveau en démographie, baptisé par Louis Henry "observation suivie" (Henry, 1963). Il y a observation suivie quand les événements vécus par un même individu ou un même couple ont été relevés à des endroits ou des moments distincts, sur des documents différant par la date, le lieu ou la nature. Ces événements sont alors classés au nom de l'individu ou du couple concerné.

Cette définition générale donne lieu à des adaptations multiples, en fonction notamment des contraintes imposées par les sources et en fonction des besolns de I'analyse. C'est ainsi qu'en vue d'étudier les comportements démographiques anciens, en particulier les comportements reproducteurs, Henry a propagé ce mode d'observation et mis au point les techniques de 
reconstitution des familles à partir des registres paroissiaux, en même temps que les procédés d'analyse qui s'y appliquent (Fleury et Henry, 1965).

Même si 1'observation suivie a d'abord et avant tout été appliquée à la reconstitution des familles, la reconstitution de populations s'est présentée comme le prolongement naturel de cette dernière. La création de fichiers de population fondés sur l'état civil - tels celui du Saguenay avec les 660000 actes de la région jusqu'en 1971 (Bouchard, 1979) ou celui du Canada pendant le régime français avec ses 300000 actes (Charbonneau et al, 1967) - s'inscrit donc dans une continuité historique marquée par la pratique de l'observation suivie.

L'unité de base de l'observation suivie, c'est la mention nominative. Dans la tradition française, il s'agit en pratique de mentions de couples à partir desquelles s'effectuent le jumelage et la reconstitution. Chaque mention de couple contient généralement les renseignements suivants : les éléments nominatifs propres aux individus concernés (prénom, patronyme et, le cas échéant, surnom); les éléments non nominatifs qui les caractérisent à ce moment (sexe, âge, état matrimonial, profession, lieu d'origine, lieu de résidence, présence ou absence); les éléments propres à l'acte lui-même (type d'acte, dates de l'acte et de l'événement correspondant - naissance, mariage ou décès -, lieu d'enregistrement); et les éléments qui situent les individus considérés par rapport aux autres personnes mentionnées dans le même acte (relation de parenté éventuelle avec le sujet de $1^{\prime}$ acte ou avec d'autres individus également mentionnés dans l'acte, degré de consanguinité entre sujets d'actes de mariage).

Ces mentions se répartissent de la manière suivante, selon que les membres du couple y apparaissent comme sujet, parent ou conjoint $^{2}$ :

M mention des sujets au mariage (au mariage du couple)

$\underline{\mathrm{Pb}}$ mention des parents de sujet d'acte de baptême (à la naissance d'un enfant)

$\underline{P m}$ mention des parents de sujet d'acte de mariage (au mariage d'un enfant)

2. Il existe également des mentions de couple témoin (par exemple la marraine d'un baptisé et son conjoint), mais ces mentions sont rarement intégrées aux reconstitutions. Elles ne le sont pas dans le cas du Saguenay. 
Ps mention des parents de sujet d'acte de sépulture (au décès d'un enfant)

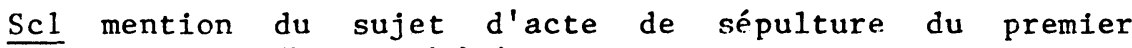
conjoint (à son décès) ainsi que du conjoint survivant

Sc2 mention du sujet d'acte de sépulture du conjoint survivant (à son décès) ainsi que du conjoint précédent déjà décédé

$\underline{\mathrm{Rm}}$ mention du sujet d'acte de mariage (au remariage du conjoint survivant) ainsi que du conjoint précédent déjà décédé.

La fiche de couple énumère en ordre chronologique toutes les mentions dans lesquelles apparaît un même couple (figure 1). La mention initiale provient normalement de l'acte de mariage (M) : c'est la date à laquelle les conjoints entrent en observation. Ils en sortent soit par décès ( $\mathrm{Scl}$ ou $\mathrm{Sc} 2$ ), soit par le remariage éventuel du conjoint survivant ( $\mathrm{Rm})$. Les enfants, pour leur part, entrent généralement en observation à leur naissance $(\mathrm{Pb})$ et ils en sortent par décès (Ps) ou par mariage $(\mathrm{Pm})$. On appelle fiche de famille une fiche de couple où les événements relatifs aux enfants ont été jumelés pour rendre compte de l'histoire de chacun de ses membres.

L'histoire individuelle des personnes ayant vécu dans un territoire donné visé par la reconstitution peut alors être déduite des informations contenues dans les fiches de famille. Dans le cas des célibataires, cette histoire tient tout entière dans une seule fiche de famille, tandis que dans le cas des personnes mariées, elle s'étale sur au moins deux fiches : celle de leur famille d'origine et celle de la famille qu'elles ont elles-mêmes fondée.

Les renseignements que renferment les histoires individuelles ou familiales obtenues des registres paroissiaux ne constituent pas en soi des histoires migratoires. Moyennant certaines hypothèses et quelques limites inévitables, ils livrent néanmoins des indications précieuses sur les trajectoires migratoires, comme nous le verrons plus loin. 
Figure 1

Exemple de fiche de couple

\begin{tabular}{|c|c|c|c|c|c|c|c|c|c|c|c|c|c|}
\hline \multicolumn{7}{|c|}{ NOM DE L'HOMME } & \multicolumn{4}{|c|}{ NOM DE LA } & \multicolumn{3}{|c|}{ FEMME } \\
\hline \multicolumn{2}{|c|}{ PILOTE } & \multicolumn{3}{|c|}{ Jakes Jimi } & \multirow[b]{2}{*}{ (F) } & \multirow[b]{2}{*}{ (G) } & \multicolumn{3}{|c|}{ DALAIRE } & \multirow{2}{*}{$\frac{\mathrm{M}}{(\mathrm{K})}$} & \multicolumn{3}{|c|}{ Demerise } \\
\hline (A) & (B) & (C) & (D) & (E) & & & $(\mathrm{H})$ & (I) & $(\mathrm{J})$ & & & （L & \\
\hline \multirow[t]{11}{*}{20149001} & 010 & 170 & 170 & 042 & 010 & 132 & 1 & 1 & M & & 11 & 09 & 1871 \\
\hline & & & & 041 & 025 & 132 & 9 & 2 & DM & 205 & 05 & 12 & 1872 \\
\hline & & & & 041 & 025 & 132 & 9 & 2 & $\mathrm{BM}$ & & 05 & 12 & 1872 \\
\hline & & & & 042 & 170 & 132 & 9 & 2 & BM & & 26 & 12 & 1873 \\
\hline & & & & 019 & 010 & 256 & 1 & 9 & DM & 516 & 04 & 05 & 1875 \\
\hline & & & & 041 & 010 & 256 & 9 & 2 & $\mathrm{BF}$ & & 21 & 02 & 1876 \\
\hline & & & & 041 & 025 & 132 & 9 & 9 & $\mathrm{DF}$ & 508 & 19 & 01 & 1877 \\
\hline & & & & 019 & 999 & 999 & 3 & 9 & D1 & 27 & 06 & 07 & 1877 \\
\hline & & & & 041 & 025 & 132 & 2 & 2 & BM & & 03 & 12 & 1877 \\
\hline & & & & 000 & 000 & & 0 & 0 & $\mathrm{R} 2$ & & 23 & 01 & 1883 \\
\hline & & & & 028 & 178 & 999 & 3 & 9 & MM & & 18 & 04 & 1898 \\
\hline
\end{tabular}

Note: Dans 1'ordre, les variables qui apparaissent dans cet exemple sont le numéro de la fiche de couple (A), le lieu de résidence du père de l'époux (B), puis du père de l'épouse (C), et enfin de l'épouse (D), la paroisse d'enregistrement (E), le lieu de résidence de l'homme (F), sa profession (G), le code de présence de l'homme (H), puis de la femme (I), le type d'événement (J), l'âge au décès s'il y a lieu (K) et la date de l'événement ( $L$ ). On trouvera le sens de chacun des codes dans SOREP (1979).

\subsection{Les données saguenayennes}

La banque de données construite au Saguenay et utilisée pour nos analyses compte 124223 actes de baptême, mariage et sépulture couvrant la période $1842-1911$ et provenant des 38 paroisses de la région existant à la fin de cette période. Ces actes ont permis de reconstituer 1 'histoire de 20106 familles ayant vécu au Saguenay jusqu'en 1911 (pour une présentation de la méthodologie relative à la reconstitution des familles, voir Bouchard, Roy et Casgrain, 1985). 
Parmi ces 20000 et quelques familles, un groupe constitué de $13 \%$ de l'ensemble a été choisi pour les fins de notre analyse : il a été établi sur la base de 15 patronymes de l'homme qui ne donnent pas lieu à des substitutions nominatives fréquentes et sont le plus souvent associés à la région de Charlevoix ${ }^{3}$. Ce second critère, en particulier, a été utilisé dans le but de faciliter les recherches ultérieures dans les répertoires de mariages ${ }^{4}$. Il est à noter que cet échantillon n'est pas représentatif de l'ensemble quant au lieu de provenance, ni vraisemblablement de la période d'arrivée des immigrants; ce défaut de représentativité ne nous paraît toutefois pas important, puisqu'il s'agit ici d'élaborer une méthode de mesure et non de caractériser l'ensemble de la population.

L'application de cette méthode suppose que la population a été correctement reconstituée, c'est-à-dire que les registres ne comportent ni lacune ni erreur, que les actes ont été correctement et intégralement dépouillés et que toutes les mentions ont bien été portées au compte de la bonne personne. Or, la qualité généra]e des informations contenues dans les registres paroissiaux saguenayens (Bourque, Markowski et Roy, 1984), de même que le soin apporté à la mise en oeuvre de la reconstitution (Bouchard, Roy et Casgrain, 1985), nous assurent de la grande qualité du corpus sur lequel repose l'élaboration de cette méthode. De plus, les opérations supplémentaires auxquelles il a été soumis dans le cadre de cette étude ont encore contribué à améliorer cette qualité générale.

3. Il s'agit des patronymes suivants : Blackburn, Boily, Bouchard, Bouliane, Brassard, Claveau, Dufour, Gobeil, Grenon, Harvey, Maltais, Martel, Pedneault, Perron et Pilote.

4. On trouvera une présentation de l'ensemble des opérations appliquées à l'échantillon dans Jetté et Gauvreau (1986a). En particulier, tous les couples dont le mariage n'avait pas été célébré au Saguenay ont fait $1^{\prime}$ objet d'une recherche dans l'ensemble des répertoires de mariages québécois et quelques-uns américains, de façon à s'assurer de leur existence et à retracer les cas les plus cachés de sous-jumelages. En général, ceux-ci devraient cependant être peu nombreux, étant donné le mode de constitution de I'échantillon. 
A la fin de ces opérations, l'échantillon de 2564 familles était prêt à servir à l'élaboration de la méthode de mesure des migrations. Il importe de préciser que cette méthode est présentée dans le but d'étudier les mouvements migratoires entre le Saguenay, région dont les registres ont été entièrement dépouillés, et l'extérieur. Les données saguenayennes contiennent également des informations précieuses quant aux mouvements à 1 'intérieur même de la région, mouvements qui ne sont pas analysés ici, mais qui pourraient être étudiés selon une méthode semblable, si par exemple les registres de quelques paroisses seulement avaient été dépouillés. Il faut donc retenir que la méthode peut s'appliquer à différents niveaux géographiques, selon les sources disponibles et les objectifs poursuivis.

\section{DEFINITION DU STATUT MIGRATOIRE}

Par définition, seuls les résidents d'une région sont susceptibles d'y entrer ou d'en sortir à un moment donné de leur existence. Ainsi, avant même de chercher à définir le statut migratoire des personnes apparaissant dans les familles reconstituées ${ }^{5}$, 11 faut identifier, puis exclure, les étrangers qui y sont mentionnés.

Ces personnes apparaissent dans les registres saguenayens soit avec une résidence toujours extérieure à la région, soit toujours comme décédées. Elles n'ont en fait jamais appartenu à la population saguenayenne : ce sont les parents d'un enfant qui se marie ici en nommant ses père et mère qui résident toujours dans une autre région ou même sont décédés, ou encore le conjoint précédent d'un veuf immigrant qui se remarie ou décède au Saguenay.

Les autres personnes sont des résidents qui peuvent être ou non des migrants. Leur statut migratoire est défini en fonction de leur naissance ou décès dans la région. Ainsi, une personne qui n'est pas née dans la région est immigrante et, de façon similaire, une personne dont le décès n'est pas enregistré dans la région est émigrante, lorsque les périodes d'observation antérfeure à la première mention et postérieure à la dernière

5. Quelques personnes migrantes qui ne sont jamais mentionnées dans les registres échappent nécessairement à cette analyse. Leur nombre est probablement restreint, et il doit surtout s'agir de personnes isolées. 
mention sont suffisamment longues. La première situation ne pose pas de problème ici puisque l'ouverture des registres coincide presque parfaitement avec le début du peuplement blanc dans la région. Dans le cas des dernières mentions, on s'est assuré ici que la personne n'apparaissait dans aucun acte pendant au moins 20 ans après sa dernière mention. Idéalement, cette période doit être la plus longue possible, à moins qu'un recensement ne serve à statuer sur la présence des individus toujours vivants en fin de période.

Les migrations dont il est question ici dans un premier temps ne concernent donc pas l'ensemble des trajectoires individuelles, mais bien le premier mouvement d'entrée d'une personne dans la région étudiée ainsi que le dernier mouvement de sortie identifiables.

On dira alors que les mentions individuelles de naissance et de décès d'une personne constituent des mentions-limites absolues par rapport à son histoire au sein du territoire observé, toutes les autres mentions par lesquelles un individu peut entrer dans la population, ou en sortir, étant qualifiées de mentions-limites relatives. Entre ces mentions-limites, 1 'hypothèse est posée que la personne réside de façon continue dans le territoire observé, à moins que l'existence d'une migration temporaire ne puisse être établie de façon certaine. Il s'agit là du principe de continuité.

Par analogie avec les histoires individuelles, les histoires familiales sont encadrées par des mentions-limites absolues ou relatives. Le mariage des parents en marque 1 'ouverture absolue tandis que le décès du second conjoint en constitue la fin irrévocable. Toute autre mention de couple correspond à une mention-limite relative familiale au delà de laquelle il y a, selon le cas, immigration ou émigration familiale.

La prise en considération des histoires familiales comporte deux avantages importants. Au nom du principe de continuité et de I'hypothèse selon laquelle le groupe familial migre ensemble tant qu'il existe ${ }^{6}$, elle autorise d'abord l'allongement de $1 \mathrm{a}$ présence des enfants membres d'une famille au delà de leurs mentions- limites individuelles. Ainsi, tant que vit sa famille et $q u^{\prime} i l$ en est membre, un individu enrichit sa fiche individuelle de toutes les mentions familiales.

6. Les enfants cessent d'appartenir à leur groupe familial au moment de leur mariage ou de leur décès, ou lorsqu'ils atteignent l'âge où ils sont en mesure de migrer seuls (voir I'Annexe A), ou encore lorsqu'ils deviennent orphelins de père et de mère. 
En second lieu, la prise en compte des histoires familiales permet de saisir une partie du phénomène des migrations temporaires, qui se traduit par des "trous" dans la trame familiale. Ces "trous" sont identifiés à l'intérieur d'une histoire familiale, par exemple par le décès ou le mariage ultérieurs d'un enfant qui n'a pas été baptisé dans la région. Ils témoignent d'une migration familiale temporaire, à moins qu'il ne s'agisse d'un acte enregistré en dehors du Saguenay avec une résidence déclarée au Saguenay (voir Jetté et Gauvreau, 1986a). Ces trous pourraient aussi résulter de lacunes dans 1 'enregistrement.

\section{DÉTERMINATION DE LA DATE DE LA MIGRATION}

Les références temporelles qui apparaissent dans les histoires individuelles ou familiales ne constituent certes pas des dates de migration (exception faite des actes de mariage qui coincideraient, par exemple, avec la migration de la femme dans la paroisse de résidence de 1 'homme). Mais elles peuvent être utilisées pour déterminer le moment de ces dernières. Il existe en effet une présomption de présence sur le territoire observé, qui s'étend plus ou moins longtemps au delà d'une mention-limite relative. Le mode de prolongation établi ici sur la base de cette hypothèse consiste justement à fixer la durée supplémentaire de présence à accorder aux individus dont l'histoire commence ou finit par une mention-limite relative. $\mathrm{Au}$ terme de la période déterminée par cette durée de prolongation, durée variable par ailleurs selon la nature de la mention, l'individu, et sa famille s'il y a lieu, est censé avoir migré.

Le mode de prolongation adopté repose sur la constatation suivante : par définition, toute mention-limite relative est précédée ou suivie immédiatement d'au moins une autre mention qui $n^{\prime}$ apparaft pas dans les registres saguenayens : c'est la mention attendue, qui peut évidemment revêtir plusieurs formes. Le moment de la migration se situe entre ces deux mentions, dans l'intervalle que nous appelons intervalle de migration.

Le calcul de la durée de cet intervalle sert de base à la détermination de la date de la migration. Il repose sur l'examen des diverses paires de mentions consécutives qui figurent dans les fiches de famille sans lacune ni migration temporaire. La fréquence relative de chaque paire de mentions est d'abord calculée, de même que la durée moyenne de l'intervalle quí sépare les deux mentions consécutives. Les tableaux 1 et 2 présentent le résultat de ces calculs. 
Tableau 1

Résultats du calcul des intervalles entre mentions consécutives constituant les fiches de couple

(ordonnées en fonction de la mention suivante attendue)

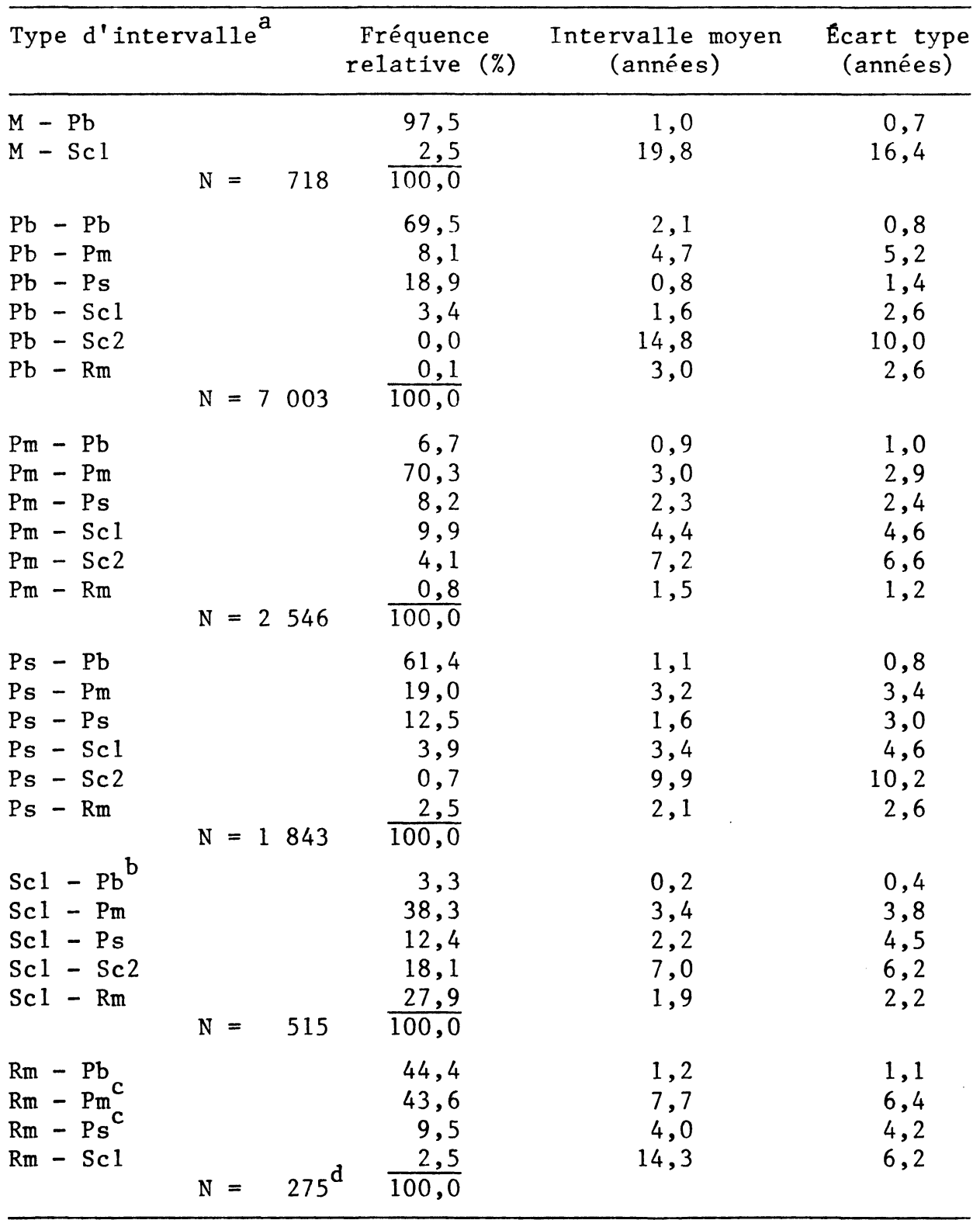

a. Pour une présentation des types de mention, voir section 1.1 .

b. Il s'agit de mentions posthumes du père décédé alors que la femme était enceinte.

c. Comme il s'agit de mentions consécutives, ces cas font référence à une mention de mariage ou de sépulture d'un enfant issu du précédent mariage.

d. Les femmes qui se remarient ne font pas partie de ce groupe, à cause du mode de sélection de l'échantillon, basé sur le patronyme de $1^{\prime}$ homme. Elles sont cependant moins nombreuses que les hommes. 
Tableau 2

Résultats du calcul des intervalles entre mentions consécutives constituant les fiches de couple (ordonnées en fonction de la mention précédente attendue)

\begin{tabular}{|c|c|c|c|c|c|c|}
\hline Type & e d'inte & $\operatorname{valle} e^{a}$ & & $\begin{array}{l}\text { Fréquence } \\
\text { relative (\%) }\end{array}$ & $\begin{array}{c}\text { Intervalle moyen } \\
\text { (années) }\end{array}$ & $\begin{array}{l}\text { Ecart type } \\
\text { (années) }\end{array}$ \\
\hline $\begin{array}{l}\mathrm{M} \\
\mathrm{Pb} \\
\mathrm{Pm} \\
\mathrm{Ps} \\
\mathrm{Scl} 1 \\
\mathrm{Rm}\end{array}$ & $\begin{array}{l}-\mathrm{Pb} \\
-\mathrm{Pb} \\
-\mathrm{Pb} \\
-\mathrm{Pb} \\
-\mathrm{Pb} \\
-\mathrm{Pb}\end{array}$ & 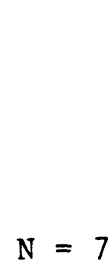 & 更 & $\begin{array}{r}10,0 \\
69,5 \\
2,4 \\
16,2 \\
0,2 \\
1,7 \\
100,0\end{array}$ & $\begin{array}{l}1,0 \\
2,1 \\
0,9 \\
1,1 \\
0,2 \\
1,2\end{array}$ & $\begin{array}{l}0,7 \\
0,8 \\
1,0 \\
0,8 \\
0,4 \\
1,1\end{array}$ \\
\hline $\begin{array}{l}\mathrm{Pb} \\
\mathrm{Pm} \\
\mathrm{Ps} \\
\mathrm{Sc} 1 \\
\mathrm{Rm}\end{array}$ & $\begin{array}{l}-P m \\
-P m \\
-P m \\
-P m \\
-P m\end{array}$ & 更 & 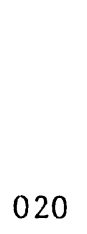 & $\begin{array}{r}18,7 \\
59,2 \\
11,6 \\
6,5 \\
4,0 \\
100,0\end{array}$ & $\begin{array}{l}4,7 \\
3,0 \\
3,2 \\
3,4 \\
7,7\end{array}$ & $\begin{array}{l}5,2 \\
2,9 \\
3,4 \\
3,8 \\
6,4\end{array}$ \\
\hline $\begin{array}{l}\mathrm{Pb} \\
\mathrm{Pm} \\
\mathrm{Ps} \\
\mathrm{Scl} 1 \\
\mathrm{Rm}\end{array}$ & $\begin{array}{l}\text { - Ps } \\
\text { - Ps } \\
\text { - Ps } \\
\text { - Ps } \\
-P s\end{array}$ & $\mathrm{~N}=1$ & 更 & $\begin{array}{r}71,4 \\
11,2 \\
12,5 \\
3,5 \\
1,4 \\
100,0\end{array}$ & $\begin{array}{l}0,8 \\
2,3 \\
1,6 \\
2,2 \\
4,0\end{array}$ & $\begin{array}{l}1,4 \\
2,4 \\
3,0 \\
4,5 \\
4,2\end{array}$ \\
\hline $\begin{array}{l}\mathrm{M} \\
\mathrm{Pb}- \\
\mathrm{Pm}- \\
\mathrm{Ps}- \\
\mathrm{Rm}-\end{array}$ & $\begin{array}{l}-S c 1 \\
-S c 1 \\
-S c 1 \\
-S c 1 \\
-S c 1\end{array}$ & $\mathrm{~N}=$ & 585 & $\begin{array}{r}3,1 \\
40,6 \\
43,0 \\
12,1 \\
1,2 \\
100,0\end{array}$ & $\begin{array}{r}19,8 \\
1,6 \\
4,4 \\
3,4 \\
14,3\end{array}$ & $\begin{array}{r}16,4 \\
2,6 \\
4,6 \\
4,6 \\
6,2\end{array}$ \\
\hline $\begin{array}{l}\mathrm{Pb} \\
\mathrm{Pm} \\
\mathrm{Ps} \\
\mathrm{Sc} 1\end{array}$ & $\begin{array}{l}-S c 2 \\
-S c 2 \\
-S c 2 \\
-S c 2\end{array}$ & $N=$ & 215 & $\begin{array}{r}1,8 \\
49,4 \\
5,5 \\
43,3 \\
100,0\end{array}$ & $\begin{array}{r}14,8 \\
7,2 \\
9,9 \\
7,0\end{array}$ & $\begin{array}{r}10,0 \\
6,6 \\
10,2 \\
6,2\end{array}$ \\
\hline $\begin{array}{l}\mathrm{Pb} \\
\mathrm{Pm} \\
\mathrm{Ps} \\
\mathrm{Scl}\end{array}$ & $\begin{array}{l}-R m \\
-R m \\
-R m \\
-R m\end{array}$ & $\mathrm{~N}=$ & $220^{d}$ & $\begin{array}{r}3,6 \\
9,5 \\
21,4 \\
65,5 \\
100,0\end{array}$ & $\begin{array}{l}3,0 \\
1,5 \\
2,1 \\
1,9\end{array}$ & $\begin{array}{l}2,6 \\
1,2 \\
2,6 \\
2,2\end{array}$ \\
\hline
\end{tabular}

Pour les notes, voir le tableau 1. 
Avant de présenter les résultats contenus dans ces tableaux, il importe de préciser un certain nombre d'éléments :

- Les deux tableaux contiennent en fait les mêmes résultats (intervalle moyen et écart type) présentés différemment, c'est-à-dire ordonnés par rapport à la dernière mentionlimite relative dans le premier cas (émigration) et à la première dans le second cas (immigration). Les intervalles ont été calculés par différence de millésime. Ce procédé est certes sommaire, mais suffisamment précis pour la méthode proposée, d'autant plus que les biais qui pourraient être introduits ont tendance à se compenser.

- Même si la banque saguenayenne utilisée ici s'étend jusqu'en 1911, seules les fiches qui s'ouvrent avant 1892 ont été considérées dans l'élaboration de ces tableaux. Le minimum de 20 années d'observation utilisé comme marge de sécurité vise à contrer deux biais : d'un côté, le risque de sélection des familles à histoire courte dans l'évaluation des intervalles généralement longs et peu fréquents (tels $\mathrm{M}-\mathrm{Scl}$ ); de l'autre, le risque de surreprésentation des intervalles généralement courts et fréquents (tels $\mathrm{Pb}-\mathrm{Pb}$ ). La limite de 20 ans qui a été retenue se justifie par le nombre peu élevé d'intervalles supérieurs à cette durée.

- Il faut se garder d'interpréter démographiquement les intervalles présentés dans ces tableaux : ainsi, l'intervalle moyen $\mathrm{Pb}-\mathrm{Pb}$ ne constitue pas un intervalle intergénésique moyen au sens démographique du terme, puisque son calcul ne fait intervenir que les cas où les deux mentions se suivent immédiatement dans $1^{\prime}$ histoire de la famille, excluant les autres, par exemple celles séparées par une sépulture.

Ces deux tableaux montrent bien que $1 \mathrm{a}$ mention attendue n'est pas unique. Seul le cas du mariage comme dernière mention-limite relative conduit à deux éventualités de mention attendue $(\mathrm{Pb}$ et $\mathrm{Scl})$, les autres conduisant généralement à au moins cinq possibilités. Par ailleurs, la fréquence des diverses paires de mentions varie considérablement pour chaque type de mention, tout comme les intervalles moyens respectifs. Ces derniers sont généralement courts : sur 29 intervalles, 10 , soit le tiers, n'atteignent même pas deux ans, tandis que 26 (87\%) sont inférieurs à dix ans. Certes, les écarts types ne sont pas négligeables, mais il importe de remarquer que les intervalles les plus longs se rapportent presque toujours aux fréquences les moins importantes. 
Dans l'ensemble, de tels résultats nous autorisent à estimer un intervalle de migration unique pour chaque type de mention relative, intervalle défini comme la moyenne pondérée des durées suivant la fréquence de chaque type de mention attendue ${ }^{7}$. En l'absence d'informations plus précises et compte tenu que les intervalles sont généralement courts, le moment de la migration est fixé à mi-chemin de la période ainsi déterminée, ce qui permet d'établir des durées de prolongation au-delà de chaque type de mention-limite relative. Ces durées de prolongation sont présentées au tableau 3.

Compte tenu des écarts types précédents, il est bien certain que les durées de prolongation obtenues ne s'appliquent pas toutes aussi parfaitement et indistinctement à toutes les situations. II s'avère cependant que les situations les plus fréquentes de mentions-limites relatives dans $l^{\prime}$ ensemble de la banque (comme le montre le tableau 4, il s'agit des mentions $\mathrm{Pb}$ et $\mathrm{Pm}$ ) sont aussi celles qui donnent lieu aux intervalles les plus sûrs, parce que courts et sujets à une moins grande variabilité ${ }^{8}$. Il est à noter que les durées de prolongation sont généralement courtes, deux d'entre elles seulement sur 12 dépassant deux années ( $\mathrm{Rm}$ et $\mathrm{Sc} 2$ ), et aucune ne dépassant quatre années.

7. Par exemple, dans le cas du mariage comme dernière mentionlimite : $(0,975 \times 1,0+0,025 \times 19,81)=1,5$ (voir tableau 1). On pourrait imaginer une application plus raffinée de ces résultats, qui tiendrait compte de divers stades dans la constitution de la famille : les résultats obtenus ne nous paraissent cependant pas exiger une aussi grande précision (à cause du regroupement de la majorité des intervalles autour de durées semblables); il ne serait de toute façon pas toujours possible, devant des familles migrantes par définition incomplètes, $d$ 'appliquer une méthode aussi raffinée.

8. Il est important de noter (pour la représentativité de 1 'échantillon utilisé ici) que cette répartition donnée pour l'ensemble de la banque est sensiblement la même que pour l'échantillon. 
Tableau 3

Durées de prolongation antérieures et postérieures correspondant aux divers types de mentions-limites relatives

\begin{tabular}{cccc}
\hline \multicolumn{2}{c}{ Prolongation postérieure } & Prolongation antérieure \\
\hline $\begin{array}{c}\text { Dernière mention } \\
\text { relative familiale }\end{array}$ & $\begin{array}{c}\text { Durée de } \\
\text { prolongation } \\
\text { (années) }\end{array}$ & $\begin{array}{c}\text { Première mention } \\
\text { relative familiale }\end{array}$ & $\begin{array}{c}\text { Durée de } \\
\text { prolongation } \\
\text { (années) }\end{array}$ \\
\hline $\mathrm{M}$ & 0,8 & $\mathrm{~Pb}$ & 0,8 \\
$\mathrm{~Pb}$ & 1,0 & $\mathrm{Pm}$ & 1,8 \\
$\mathrm{Pm}$ & 1,6 & $\mathrm{Ps}$ & 0,6 \\
$\mathrm{Ps}$ & 0,9 & $\mathrm{Scl}$ & 1,9 \\
$\mathrm{Scl}$ & 1,7 & $\mathrm{Rm}$ & 1,0 \\
$\mathrm{Rm}$ & 2,3 & $\mathrm{Sc} 2$ & 3,8 \\
\hline
\end{tabular}

Tableau 4

Répartition des familles de l'ensemble de la banque selon les mentions-limites relatives

\begin{tabular}{crrcrrr}
\hline $\begin{array}{c}\text { Dernière mention- } \\
\text { limite relative }\end{array}$ & No & $\%$ & $\begin{array}{c}\text { Première mention- } \\
\text { limite relative }\end{array}$ & No & $\%$ \\
\hline $\mathrm{M}^{\mathrm{a}}$ & 339 & 11,9 & $\mathrm{~Pb}$ & 1807 & 53,2 \\
$\mathrm{~Pb}$ & 1060 & 37,1 & $\mathrm{Pm}$ & 976 & 28,7 \\
$\mathrm{Pm}$ & 736 & 25,7 & $\mathrm{Ps}$ & 269 & 7,9 \\
$\mathrm{Ps}$ & 351 & 12,3 & $\mathrm{Sc1}$ & 138 & 4,1 \\
$\mathrm{Scl}$ & 371 & 13,0 & $\mathrm{Rm}$ & 96 & 2,8 \\
& & & $\mathrm{Sc} 2$ & 113 & 3,3 \\
\hline Ensemble & 2857 & 100,0 & Ensemble & 3 & 399 & 100,0 \\
\hline
\end{tabular}

a. Cette catégorie comprend ici les premiers marlages de même que les mariages de rang supérieur à un, ce qui explique l'absence de mentions de type $\mathrm{Rm}$. 
Le mode général de prolongation présenté ici, qui s'appuie sur les cas connus pour déterminer des durées applicables aux cas inconnus, n'est évidemment acceptable que s'il n'existe pas de différence significative entre les deux groupes de cas, qui sont essentiellement les familles sédentaires et celles qui migrent. Cette hypothèse se subdivise elle-même en deux hypothèses secondaires, la première étant que les comportements démographiques des deux groupes ne diffèrent pas, et la seconde étant que la répartition des divers types de mentions attendues dans les cas connus s'applique bien aux cas inconnus, c'est-àdire qu'il n'y a pas de sélection particulière dans le moment de la migration en rapport avec cette répartition.

I1 n'est pas facile de statuer sur la première hypothèse, que peu d'études ont spécifiquement abordée (voir Henry, 1976). On sait par ailleurs que les personnes migrantes connaissent en général des niveaux de mortalité plus faibles que les non migrantes, à cause d'une sélection qui s'opère en faveur des individus les plus "robustes". D'autres types de sélection sont possibles, par exemple en fonction de l'âge au mariage, les migrants se mariant souvent à un âge plus tardif, ce qui peut en retour affecter la dimension des familles ainsi formées (Gauvreau, 1986). Cependant, comme les calculs effectués ici concernent également des familles migrantes à un niveau intra-régional et qu'ils incluent les mentions consécutives des familles antérieurement ou ultérieurement migrantes, le biais éventuel occasionné par cette différence devrait être mineur.

Quant à la seconde hypothèse, elle ne peut conduire qu'à des erreurs d'estimation minimes des durées de prolongation. Par exemple, même si les mentions-limites relatives de type $\mathrm{Pb}$ étaient moins souvent suivies d'un décès d'enfant que ne le sont les cas connus, parce que ce décès suivrait de peu l'acte de baptême, transformant du coup la mention-limite relative de type $\mathrm{Pb}$ en $\mathrm{Ps}$, la durée de prolongation ne serait pas modifiée de façon substantielle. Cette situation résulte des répartitions spécifiques observées suivant le type des mentions-limites relatives (voir tableaux 1 et 2 ).

I1 faut également préciser que les durées spécifiques de prolongation obtenues ici pour déterminer les dates de migration ne sont valables en principe que dans le cadre saguenayen où elles ont été élaborées. Toutefois, elles pourraient vraisemblablement être étendues à toute population présentant un comportement démographique semblable. 
Les résultats précédents ne concernent que les situations de mentions-limites familiales. Il existe encore des situations de premières mentions relatives individuelles d'une personne à son mariage ou à son décès, ou de dernière mention relative d'une personne à sa naissance, qui n'apparaissent pas dans les résultats précédents. La dernière situation renvoie à la question de l'âge à partir duquel un individu peut migrer seul et ne doit plus être considéré comme membre de sa famille d'origine : elle est traitée séparément à $l^{\prime}$ annexe $A$, qui suggère que cet âge se situe autour de 15 ans. Les deux premières situations posent quant à elles la question du temps passé en territoire saguenayen avant qu'un individu s'y marie ou y décède. Le jumelage des individus concernés avec les données des recensements nous apprend tout au plus que cette durée peut aller jusqu'à cinq ans dans le cas des mariages, bien qu'elle se situe autour de trois ans en moyenne; elle est probablement plus courte dans le cas des décès.

\section{LA VALIDATION DU MODE DE PROLONGATION}

L'évaluation d'une méthode de mesure de la migration à partir de mentions à l'état civil devrait reposer sur le recours à des enregistrements de mouvements migratoires. C'est faute d'en disposer qu'on est contraint de se rabattre sur un test moins direct et moins précis, le recours aux mentions nominatives tirées des trois recensements fédéraux de 1852, 1861 et 1871 (pour une présentation de ces recensements, voir Pouyez et alii, 1983). Ce test repose sur le jumelage des familles appartenant à l'échantillon avec les ménages recensés lors des trois premiers recensements nominatifs régionaux (pour une présentation de cette opération de jumelage, voir Jetté et Gauvreau, 1986b). I1 a pour but de vérifier la valeur du mode de prolongation pour déterminer les dates de migration, en même temps qu'il fournit une estimation du nombre de personnes migrantes qui échappent à l'étude fondée sur les familles reconstituées.

Pour le réaliser, on a extrait de l'échantillon les fiches commençant par une première mention-limite relative entre le ler janvier 1852 et le 31 décembre 1891, c'est-à-dire entre l'année du premier recensement nominatif et 20 ans après le dernier. De même, on a extrait les fiches se terminant par une dernière mention-limite relative entre le ler janvier 1842 et le 31 décembre 1871 , c'est-à-dire depuis $1^{\prime}$ ouverture des registres jusqu'à $I^{\prime}$ année du dernier recensement nominatif. 
Les fiches de chacun des deux groupes ont été distribuées selon les caractéristiques suivantes : le type de mention-limite relative, le fait que la famille apparaisse ou non au recensement le plus proche de cette mention (précédant la première, ou suivant la dernière), ainsi que la durée (calculée par différence de millésime) entre la mention et le moment du recensement. Les tableaux 5 et 6 présentent les résultats de cette double répartition. On notera qu'on a exclu du premier tableau le cas des fiches commençant par une première mention de type $\mathrm{Pb}$, $\mathrm{Ps}$ ou $\mathrm{Rm}$ dont la date du mariage, célébré hors Saguenay, suivait le recensement avec lequel on voulait les comparer : ces familles n'ont évidemment pas été recensées, ce qui dans tous les cas s'accordait avec le résultat de l'application de la règle de prolongation.

Comme les recensements disponibles appartiennent tous aux premières décennies, et que l'étude ne porte que sur un échantillon, il est clair que le test effectué ici se heurte rapidement au problème des petits nombres. Il en ressort toutefois qu'une majorité de personnes migrantes n'ont pas été recensées au recensement précédant leur première mention dans les registres (tableau 5) ou suivant leur dernière mention (tableau 6). Cette situation vaut particulièrement dans le cas des mentions $\mathrm{Pb}$ et $\mathrm{Pm}$, qui sont les plus nombreuses ici, de même que parmi les cas auxquels les durées de prolongation doivent être appliquées.

De plus, lorsque des personnes migrantes sont recensées, c'est en général à la durée 0 , c'est-à-dire lorsque cette mention est enregistrée l'année même du recensement, ou encore à la durée 1 , ce qui s'accorde tout à fait avec les résultats précédents. Certes, on introduit un certain nombre d'erreurs en fixant le moment de la migration aux dates uniformes calculées au tableau 3, mais ces erreurs sont relativement faibles en termes absolus. Comme on pouvait s'y attendre, et malgré les faibles effectifs de ces catégories, elles sont toutefois plus importantes pour les types de mentions sujets à des intervalles entre mentions successives de durée plus variable (tableaux 1 et 2).

En rapport avec l'exhaustivité de la mesure des migrations établie à partir des familles reconstituées, le jumelage des recensements avec les familles fatt par ailleurs apparaftre un certain nombre de personnes recensées qui n'apparaissent jamais dans les familles reconstituées. Ces personnes sont peu nombreuses, comptant pour environ $5 \%$ des recensées : 11 n'y a que deux couples parmi elles, toutes les autres étant des personnes isolées, enfants ou jeunes adultes dont le séjour au Saguenay n'a été marqué par aucune mention dans les registres. 
Tableau 5

Présence au recensement précédent selon le type de la première mention relative et la durée écoulée entre cette mention et le recensement

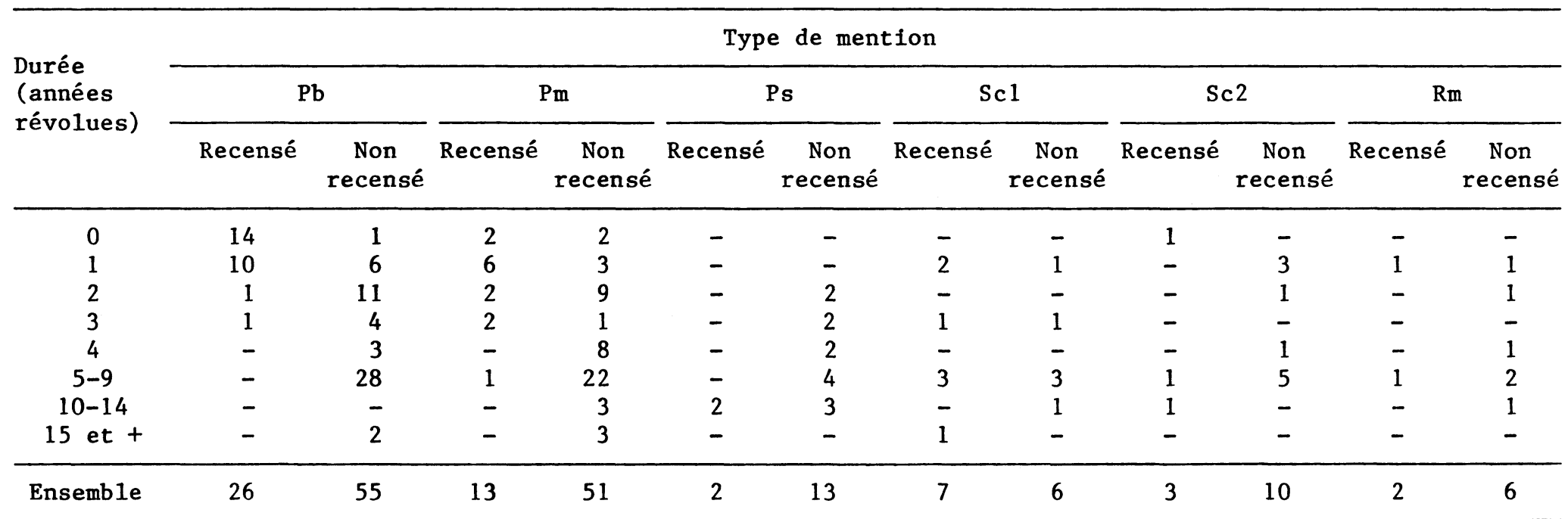


Tableau 6

Présence au recensement suivant selon le type de la dernière mention relative et la durée écoulée entre cette mention et le recensement

\begin{tabular}{|c|c|c|c|c|c|c|c|c|c|c|}
\hline \multirow{3}{*}{$\begin{array}{l}\text { Durée } \\
\text { (années } \\
\text { révolues) }\end{array}$} & \multicolumn{10}{|c|}{ Type de mention ${ }^{a}$} \\
\hline & \multicolumn{2}{|c|}{ M } & \multicolumn{2}{|c|}{$\mathrm{Pb}$} & \multicolumn{2}{|c|}{$\mathrm{Pm}$} & \multicolumn{2}{|c|}{$\mathrm{Ps}$} & \multicolumn{2}{|c|}{$\mathrm{Sc} 1$} \\
\hline & Recensé & $\begin{array}{l}\text { Non } \\
\text { recensé }\end{array}$ & Recensé & $\begin{array}{l}\text { Non } \\
\text { recensé }\end{array}$ & Recensé & $\begin{array}{c}\text { Non } \\
\text { recensé }\end{array}$ & Recensé & $\begin{array}{l}\text { Non } \\
\text { recensé }\end{array}$ & Recensé & $\begin{array}{l}\text { Non } \\
\text { recensé }\end{array}$ \\
\hline 0 & - & - & 4 & - & 1 & - & - & - & 1 & - \\
\hline 1 & - & - & 5 & - & 2 & - & - & - & - & 2 \\
\hline 2 & 1 & - & 2 & 5 & - & 1 & - & - & 1 & - \\
\hline 3 & 1 & 2 & - & 1 & - & 2 & - & 1 & - & 1 \\
\hline 4 & - & - & - & - & 2 & 1 & 1 & - & 1 & - \\
\hline $5-9$ & 1 & 1 & - & 7 & 1 & 8 & 1 & 3 & - & 1 \\
\hline $10-14$ & - & - & - & - & 1 & - & - & - & - & - \\
\hline Ensemb1e & 3 & 3 & 11 & 13 & 7 & 12 & 2 & 4 & 3 & 4 \\
\hline
\end{tabular}

a. II n'y a aucun cas de type $\mathrm{Rm}$. 
Signalons par ailleurs que le phénomène des personnes mentionnées dans les registres mais n'apparaissant à aucun recensement est par opposition beaucoup plus fréquent et n'affecte pas que des personnes isolées. Ce résultat met en évidence certaines limites inhérentes à la mesure du phénomène migratoire à partir d'une comparaison entre recensements distants par exemple de quelques années.

Dans l'ensemble, le test réalisé à l'aide des mentions aux recensements nominatifs confirme donc le caractère quasi exhaustif de la méthode qui vient d'être proposée et qui est basée sur les familles reconstituées. Seul un certain nombre de personnes isolées, qui n'ont fait qu'un bref séjour dans la région, y échappent inévitablement. Le test confirme également que les mouvements d'entrée et de sortie respectivement précèdent ou suivent de peu la mention-limite relative apparaissant dans les registres. Malgré certaines limites liées à la plus grande variabilité de certains intervalles, le mode de prolongation proposé et les durées de prolongation qui en découlent constituent donc de bons outils pour fixer le moment où sont effectuées les migrations.

\section{CONCLUSION}

Devant la difficulté d'obtenir des mesures et une analyse appropriées des mouvements migratoires au sein des populations du passé, la possibilité d'avoir recours aux histoires familiales et individuelles tirées de la reconstitution des familles ouvre une voie importante pour l'étude du phénomène migratoire. Comme on vient de le montrer, un traitement adéquat des informations rassemblées sous cette forme permet en effet d'étudier l'ampleur et le calendrier des migrations.

Deux aspects de cette méthode paraissent surtout importants. Le premier tient au fait qu'elle permet d'appréhender séparément, mais en offrant la possibilité de les combiner sur une base individuelle, les mouvements d'immigration et d'émigration. Certes, tous les mouvements d'aller-retour ne peuvent être saisis par cette méthode, mais une majorité des entrées et des sorties le sont vraisemblablement. 
Le second aspect concerne la possibilité de fixer avec une bonne précision le moment de ces divers mouvements. En effet, dans le contexte d'un régime démographique de très forte fécondité et de mortalité assez élevée, le nombre important de mentions que regroupe chaque fiche de famille permet de statuer sur le moment d'entrée dans la région ou celui de la sortie. Le premier précède en général de peu le moment de la première apparition dans les registres, tandis que le second suit également de peu le moment de la dernière apparition, comme le confirme généralement le test de validation effectué à partir des recensements.

Sur la base de ces informations, une caractéristique plus complète du processus migratoire dans l'espace et dans le temps est désormais possible, de même qu'une analyse plus fine de certaines de ses caractéristiques (âge au moment de la migration, stade de constitution de la famille, relations de parenté entre personnes migrantes, etc.). Ces éléments sont essentiels pour la compréhension de la mise en place et du mode de formation des populations du passé. Ils le sont d'autant plus dans le cas d'une région comme le Saguenay au cours du XIXe siècle, tant d'un point de vue historique que démographique ou génétique. 
ANNEXE A

L'ÁGE-LIMITE INFÉRIEUR AUQUEL UN INDIVIDU PEUT MIGRER SEUL

Le principe selon lequel les membres d'une famille migrent ensemble connât nécessairement certaines limites qui sont liées au fait que les enfants deviennent progressivement autonomes par rapport à leur famille d'origine. Leurs déplacements obéissent alors de moins en moins à un tel principe : ils peuvent migrer seuls sans leur famille, tout comme ils peuvent demeurer dans la région alors que leur famille en sort. La question qui nous intéresse ici est de savoir à partir de quel âge se manifeste cette relative autonomie des enfants par rapport à leur famille.

Une réponse à cette question peut être obtenue en utilisant les données des trois recensements de 1852,1861 et 1871 d'une façon semblable à ce qui a été fait pour la validation des règles de prolongation. Nous appuyant sur le jumelage des individus appartenant aux familles de l'échantillon avec les membres des ménages recensés, nous avons d'abord examiné le cas des enfants dont la seule mention dans les registres saguenayens est celle correspondant à leur naissance entre le ler janvier 1842 et le 31 décembre 1870, pour savoir s'ils étaient bien recensés avec leur famille aux recensements suivant cette mention. L'information a été répartie selon l'âge de ces enfants au moment du recensement (tableau A-1).

Bien que 1'exhaustivité des registres et des recensements puisse également être en cause pour expliquer les proportions observées d'enfants non recensés, 11 paraît assez clair que celles-ci augmentent avec l'âge (11 n'y a pas de différence nette à cet égard entre les filles et les garçons). Ce résultat général renforce l'hypothèse selon laquelle 11 existe un âge au delà duquel la migration individuelle doit être admise. Dès le second groupe quinquennal d'âge, une certaine propension à migrer seul apparait, propension qui devient surtout manifeste après 15 ans. Cette impression est par ailleurs confirmée par les données concernant les enfants de destin connu, solt ceux qui se marient ou décèdent par la suite au Saguenay (tableau A-2). Pour eux, la migration n'aura été que temporaire. Comme on pouvait $s^{\prime} y$ attendre, le phénomène semble nettement plus fréquent chez les orphelins, quel que soit leur âge. 
$\mathrm{Au}$ vu de ces résultats, on admettra donc qu'un enfant peut migrer seul dès l'âge de cinq ans, que la probabilité qu'il le fasse augmente avec son âge à la dernière mention familiale et, enfin, que cette probabilité devient surtout significative à partir de 15 ans. En pratique, dans le cas qui nous intéresse, nous considérons que le principe de la migration familiale cesse de s'appliquer aux enfants lorsqu'ils atteignent l'âge de 15 ans.

Tableau A-1

Proportion d'enfants non recensés parmi ceux qui n'apparaissent dans les registres qu'à leur baptême, suivant l'âge de l'enfant au moment du recensement

\begin{tabular}{ccc}
\hline $\begin{array}{l}\text { Groupe } \\
\text { d'âge }\end{array}$ & $\begin{array}{c}\text { Nombre } \\
\text { de cas }\end{array}$ & $\begin{array}{c}\text { Proportion d'enfants } \\
\text { non recensés }\end{array}$ \\
\hline $0-4$ & 231 & $1,7 \%$ \\
$5-9$ & 145 & $7,6 \%$ \\
$10-14$ & 98 & $4,1 \%$ \\
$15-19$ & 30 & $13,3 \%$ \\
$20-24$ & 15 & $33,3 \%$ \\
$25-29$ & 4 & - \\
Ensemble & 523 & $5,4 \%$ \\
\hline
\end{tabular}

a. Un même enfant peut être compté dans plus d'un groupe d'âge si plus d'un recensement a suivi sa mention de baptisé. 
Tableau A-2

Proportion d'enfants non recensés au Saguenay ou recensés hors de leur famille, parmi ceux de destin connu ${ }^{a}$, selon I'âge de I'enfant au moment du recensement

\begin{tabular}{cccc}
\hline $\begin{array}{l}\text { Groupe } \\
\text { d'âge }\end{array}$ & $\begin{array}{c}\text { Nombre de cas } \\
\text { de destin connu }\end{array}$ & $\begin{array}{c}\text { Proportion d'enfants } \\
\text { non recensés } \\
\text { au Saguenay }\end{array}$ & $\begin{array}{c}\text { Proportion d'enfants } \\
\text { recensés au Saguenay } \\
\text { hors de leur famille }\end{array}$ \\
\hline $0-4$ & 584 & - & - \\
$5-9$ & 470 & $2,6 \%$ & $0,6 \%$ \\
$10-14$ & 346 & $2,4 \%$ & $2,3 \%$ \\
$15-19$ & 208 & $4,5 \%$ & $4,3 \%$ \\
$20-24$ & 89 & $6,6 \%$ & $11,2 \%$ \\
$25-29$ & 15 & $1,2 \%$ & - \\
Ensemble & 1512 & & $1,8 \%$ \\
\hline
\end{tabular}

a. C'est-à-dire qui sont nés au Saguenay et qui s'y marient ou y décèdent par la suite.

b. Un même enfant peut être compté dans plus d'un groupe d'âge si plus d'un recensement a suivi sa mention de baptisé. 
BOUCHARD, Gérard, 1979. "Un essai d'anthropologie régionale : l'histoire sociale du Saguenay au XIXe et XXe siècles". Annales Économie-Sociétés-Civilisations, 1, 106-125.

BOUCHARD, Gérard et André LAROSE, 1979. "La réglementation du contenu des actes de baptême, mariage, sépulture au Québec, des origines à nos joursh. Revue d'histoire de 1'Amérique française, $30,1,67-84$.

BOUCHARD, Gérard, Raymond ROY et Bernard CASGRAIN, 1985. Reconstitution automatique des familles. Le système SOREP. Chicoutimi, Université du Québec à Chicoutimi, SOREP, Dossier no 2,2 volumes, 521 et 224 pages.

BOURQUE, Mario, France MARKOWSKI et Raymond ROY, 1984. "Evaluation du contenu des registres de l'état civil saguenayens, 1842-1951". Archives, 16, 3, 16-39.

CHARBONNEAU, Hubert, Jacques LÉGARE, René DUROCHER, Gilles PAQUET et Jean-Pierre WALLOT, 1967. "La démographie historique au Canada : un projet de recherchel. Recherches sociographiques, $8,2,214-217$.

CRAIG, Béatrice, 1983. Migrant Integration and Kinship Ties in a Frontier Community. Paper presented at the 1983 annual meeting of the Canadian Historical Association, Vancouver.

FLEURY, Michel et Louis HENRY, 1965. Nouveau manuel de dépouillement et d'exploitation de l'état civil ancien. Paris, Institut National d'Études Démographiques, 176 pages.

GAUVREAU, Danielle, 1986. Reproduction humaine et reproduction sociale : la ville de Québec pendant le régime français. Montréal, Université de Montréal, thèse de doctorat en démographie, 442 pages.

HENRY, Louis, 1963, "Réflexions sur l'observation en démographien. Population, 18, 2, 235-262.

HENRY, Louis, 1976. "Mobilité et fécondité d'après les fiches de familles". Annales de démographie historique, 279-304. 
JETTE, René, 1980. Reconstitution de recensements à partir de registres paroissiaux : analyse méthodologique sur échantillon. Montréal, Université de Montréal, thèse de doctorat en démographie, 332 pages.

JETTÉ, René et Danielle GAUVREAU, 1986a. En marge de l'élaboration d'une méthode de mesure des migrations à partir des familles reconstituées. Chicoutimi, Université du Québec à Chicoutimi, SOREP, document no II-C-121, $15 \mathrm{p}$.

JETTE, René et Danielle GAUVREAU, 1986b. Le processus de validation du mode de prolongation utilisé dans 1'étude des migrations : le jumelage des recensements aux familles reconstitués. Chicoutimi, Université du Québec à Chicoutimi, SOREP, document no II-C-122, 7 p.

PARKERSON, Donald H., 1982. "How mobile were nineteenth-century Americans?". Historical Methods, 15, 3, 99-109.

POUYEZ, Christian et Miche1 BERGERON, 1978. "L'étude des migrations au Saguenay (1842-1931) : problèmes de méthodel. Histoire sociale/Social History, $11,21,26-61$.

POUYEZ, Christian, Yolande LAVOIE, Gérard BOUCHARD, Raymond ROY et alii, 1983. Les Saguenayens. Introduction à l'histoire des populations du Saguenay, 16e - 20e s. Québec, Presses de 1'Université du Québec, 510 pages.

POUYEZ, Christian, Raymond ROY et Gérard BOUCHARD, 1981. "La mobilité géographique en milieu rural : le Saguenay, 1852-1861". Histoire sociale/Social History, 14, 27, 123-155.

POUYEZ, Christian, Raymond ROY and François MARTIN, 1982. "The Linkage of Census Name Data : Problems and Procedures". Journal of Interdisciplinary History, 14, 1, 129-152.

SOREP, 1979. Manuel d'instruction pour le développement des registres de baptêmes, mariages et sépultures. Chicoutimi, Université du Québec à Chicoutimi, document II-C-3, 92 p.

WATKINS, Susan C. and Myron P. GUTMANN, 1983. kMethodological Issues in the Use of Population Registers for Fertility Analysis". Historical Methods, 16, 3, 109-120. 


$$
\text { RÉSUME - SUMMARY - RESUMEN }
$$

JETTE René et GAUVREAU Danielle - DES FICHES DE FAMILLE A LA MESURE DES MIGRATIONS : UNE MÉTHODE ÉLABORÉ A PARTIR DES DONNEES DU SAGUENAY AU XIXe SIECLE

I1 n'est pas facile de mesurer ni de caractériser les mouvements migratoires, encore moins quand ils concernent les populations du passé. Le présent texte propose à cet effet une méthode inédite fondée sur les famllles reconstituées à partir des registres paroissiaux saguenayens. Utilisant les biographies individuelles et familiales, cette méthode permet d'étudier séparément les mouvements d'immigration et d'émigration. Dans le cas du Saguenay, et probablement de toute autre région à fécondité et mortalité élevées, elle permet par ailleurs de fixer la date des migrations à un moment peu éloigné de la première ou de la dernière apparition dans le fichier des familles.

JETTE René and GAUVREAU Danielle - MEASURING MIGRATION FROM FAMILY FILES : A METHOD BASED ON DATA FOR THE SAGUENAY REGION IN THE 19th CENTURY

Measuring migration is not an easy task, particularly in historical demography. This paper uses a new approach, based on families reconstituted from the Saguenay parish registers. Making use of the individual and family biographies, this method allows for a separate analysis of inmigration and outmigration. In the case of Quebec's Saguenay region, and probably in the case as well of any region experiencing high fertility and mortality rates, the method allows also to determine the date of migration at a moment which is close to the first or the last entering in the family file. 
JETTE René y GAUVREAU Danielle - MEDIR LAS MIGRACIONES UTILIZÁNDO LAS FICHAS DE FAMILIA : UN MÉTODO ELABORADO A PARTIR DE DATOS DEL SAGUENAY EN EL SIGLO XIX

No es fácil de medir ni de caracterizar los movimientos migratorios y aún menos cuando se trata de poblaciones del pasado. Este texto propone para ello un método inédito basado sobre la reconstitución de las familias, a partir de los registros parroquiales del Saguenay. Utilizando las biografias individuales y familiales, este método permite estudiar separadamente los movimientos de inmigración y de emigración. En el caso del Saguenay y posiblemente en cualquier otra región con una fecundidad y una mortalidad elevadas, éste permite además determinar la fecha de las migraciones en un momento no muy lejano de la primera o ültima inscripción en el fichero de las familias. 